Aims. This study examines the prevalence and associations between recent violence experience, mental health and physical health impairment among Female Sex Workers (FSWs) in north Karnataka, India. Background. Multi-morbidity, in particular the overlap between physical and mental health problems, is an important global health challenge to address. FSWs experience high levels of gender-based violence, which increases the risk of poor mental health, however there is limited information on the prevalence of physical health impairments and how this interacts with mental health and violence. Method. We conducted secondary analysis of cross-sectional quantitative survey data collected in 2016 as part of a cluster-RCT with FSWs called Samvedana Plus. Bivariate and multivariate analyses were used to examine associations between physical impairment, recent (past 6 months) physical or sexual violence from any perpetrator, and mental health problems measured by PHQ-2 (depression), GAD-2 (anxiety), any common mental health problem (depression or anxiety), self-harm ever and suicidal ideation ever.

Result. 511 FSWs participated. One fifth had symptoms of depression $(21.5 \%)$ or anxiety $(22.1 \%)$, one third $(34.1 \%)$ reported symptoms of either, $4.5 \%$ had ever self-harmed and 5.5\% reported suicidal ideation ever. Over half (58.1\%) reported recent violence. A quarter (27.6\%) reported one or more chronic physical impairments. Mental health problems such as depression were higher among those who reported recent violence (29\%) compared to those who reported no recent violence $(11 \%)$. There was a step-wise increase in the proportion of women with mental health problems as the number of physical impairments increased (e.g. depression $18.1 \%$ no impairment; $30.2 \%$ one impairment; $31.4 \% \geq$ two impairments). In adjusted analyses, mental health problems were significantly more likely among women who reported recent violence (e.g. depression and violence AOR 2.42 (1.24-4.72) with rates highest among women reporting recent violence and one or more physical impairments (AOR 5.23 (2.49-10.97).

Conclusion. Our study suggests multi-morbidity of mental and physical health problems is a concern amongst FSWs and is associated with recent violence experience. Programmes working with FSWs need to be mindful of these intersecting vulnerabilities, inclusive of women with physical health impairments and include treatment for mental health problems as part of core-programming.

Samvedana Plus was funded by UKaid through Department for International Development as part of STRIVE (structural drivers of HIV) led by London School of Hygiene and Tropical Medicine and the What Works to Prevent Violence Against Women and Girls Global Programme led by South African Medical Research Council

\section{Prevalence and correlates of common mental health problems and recent suicidal behaviour among female sex workers in Nairobi, Kenya: findings from the Maisha Fiti study}

Alicja Beksinska ${ }^{1 \star}$, Zaina Jama ${ }^{2}$, Rhoda Kabuti², Mary Kungu², Hellen Babu ${ }^{2}$, Emily Nyakiri ${ }^{2}$, Pooja Shah ${ }^{1}$, Chrispo Nyabuto ${ }^{2}$, Monica Okumu², Anne Mahero ${ }^{2}$, Pauline Ngurukiri², Erastus Irungu ${ }^{2}$, Wendy Adhiambo ${ }^{2}$, Peter Muthoga ${ }^{2}$, Rupert Kaul ${ }^{3}$, Janet Seeley ${ }^{1}$, Tara S Beattie ${ }^{1}$, Joshua Kimani ${ }^{2}$ and Helen Weiss ${ }^{4}$

${ }^{1}$ Department of Global Health and Development, London School of Hygiene and Tropical Medicine; ${ }^{2}$ UK Partners for Health and Development in Africa (PHDA), University of Nairobi Institute of Tropical and Infectious Diseases; ${ }^{3}$ University of Toronto and ${ }^{4} \mathrm{MRC}$ International Statistics \& Epidemiology Group, Department of Infectious Disease Epidemiology, London School of Hygiene and Tropical Medicine

${ }^{*}$ Corresponding author.

doi: 10.1192/bjo.2021.637
Aims. Adverse childhood experiences (ACEs), poverty, violence and harmful alcohol/substance are associated with poor mental health outcomes in the general population. These risks are likely to be exacerbated among Female Sex Workers (FSWs), however there are few studies examining risks factors for mental health problems among FSWs. We examine the prevalence and correlates of common mental health problems including suicidal behaviour among FSWs in Kenya.

Method. Maisha Fiti is a longitudinal study among FSWs randomly selected from Sex Worker Outreach Programme (SWOP) clinics across Nairobi. Baseline data were collected from June-December 2019. Mental health problems were assessed using the Patient Health Questionnaire (PHQ-9) for depression, the Generalised Anxiety Disorder tool (GAD-7) for anxiety, and the Harvard Trauma Questionnaire (HTQ-17) for PostTraumatic Stress Disorder (PTSD). Recent suicidal behaviour was defined as reported suicide attempt or suicidal ideation in the past 30 days. Other measurement tools included the WHO Adverse Childhood Experiences (ACE) score, WHO Violence Against Women questionnaire, and the WHO ASSIST tool (to measure harmful alcohol/substance use in the past 3 months). Descriptive statistics and multivariable logistic regression were conducted in Stata 16.1.

Result. Of 1039 eligible FSWs, 1003 FSWs took part in the study (response rate: 96\%) with a mean age of 33.7 years. The prevalence of moderate/severe depression was 23.2\% (95\%CI: $20.7-$ $25.9 \%$ ), moderate/severe anxiety $11.0 \%$ (95\%CI: 9.3-13.1\%), PTSD 14.0\% (95\% CI: 12.2-16.5\%) and recent suicidal behaviour $10.2 \%$ (95\%CI: $8.5-12.2 \%$ ) (2.6\% suicide attempt; $10.0 \%$ suicidal ideation). Among women with any mental health problem $63.0 \%$ also had a harmful alcohol/substance use problem. One in four women (25\%; 95\%CI: 22.5-27.8\%) had depression and/or anxiety and this was independently associated with higher ACE scores, hunger (skipped a meal in last week due to financial difficulties), death of a child, perceived sex work stigma and recent sexual/physical violence. PTSD was associated with higher ACE scores, hunger, increased STI prevalence (chlamydia trachomatis) and recent violence. Recent suicidal behaviour was associated with higher ACE scores, low literacy, hunger, and recent violence. Mental health problems and suicidal behaviour were less prevalent among women reporting social support.

Conclusion. The high burden of mental problems among FSWs indicates a need for accessible services tailored for FSWs alongside broader structural interventions addressing poverty, harmful alcohol/substance use and violence. High rates of ACEs among this population indicates the need to consider early childhood and family interventions to prevent poor mental health outcomes.

Funding: Medical Research Council and the UK Department of International Development

\section{Prevalence of psychological stress and adopted coping strategies among healthcare workers in King Saud Medical City (KSMC)}

\author{
Mohammed Binnwejim* and Atheer Alhumade \\ Imam Muhammed Bin Saud Islamic University \\ ${ }^{\star}$ Corresponding author.
}

\section{doi: 10.1192/bjo.2021.638}

Aims. The present study aims at investigating the level of stress among Saudi healthcare workers during COVID-19 pandemic. In addition, the present study sought to identify the coping 
strategies adopted by Saudi healthcare workers to alleviate the stress related to COVID-19 pandemic.

Method. A descriptive cross-sectional study was performed in the period between September and November/2020. A sample of 381 healthcare workers (Physicians, nurses, and technicians) were recruited from King Saud Medical City (KSMC). Both Perceived Stress Scale-4 (PSS-4) and BRIEF-COPE scale were used to assess the levels of stress and the stress coping strategies, respectively. Descriptive statistics were used to analyze the healthcare workers' responses about the COVID-19 related stress and their adopted coping strategies

Result. The results of the study showed that there was a moderate to high level of COVID-19 related stress $(11.64 \pm 0.73)$ among the Saudi healthcare workers. In addition, it was found that planning $(3.89 \pm 0.61)$, positive reframing (3.69 \pm 0.77$)$, venting (3.39 \pm $1.01)$, and emotional support $(3.27 \pm 0.63)$ were the most adopted coping strategies by the healthcare workers to overcome and reduce the stress levels

Conclusion. The study concluded that both problem-focused and emotion-focused stress coping strategies were the most commonly adopted coping strategies among Saudi healthcare workers in KSMC. The study recommends increasing the number of the healthcare workers in the KSMC, in addition to increasing the healthcare workers' knowledge, awareness and practice of the stress coping strategies, especially in crisis events, such as COVID-19 pandemic.

\section{Psychiatric comorbidities with autism spectrum disorder in an adult clinic sample}

Rifat Binte Radwan ${ }^{1 \star}$ and Chiro Islam Mallik ${ }^{2}$

${ }^{1}$ Royal Victoria Infirmary, Cumbria, Northumberland, Tyne and Wear NHS Foundation trust and ${ }^{2}$ Community Learning Disability Team, Hertfordshire Partnership University NHS Foundation Trust ${ }^{*}$ Corresponding author.

doi: 10.1192/bjo.2021.639

Aims. As part of continuity, prevalence of Autism Spectrum Disorder (ASD) is nearly the same in adults as children and is associated with other comorbid psychiatric disorders that have substantial impact on their life and complex the intervention. This study aimed to examine psychiatric comorbidity in referred adult ASD patients compared to non-ASD psychiatric patients. It has been hypothesized that comorbid psychiatric disorders were higher among patients with ASD than patients without ASD.

Method. In total, 36 adults with ASD referred in the year 2019 in a psychiatric consultation center in Dhaka city were included in the study. They were derived from the case register of the center. Similar number of age and sex-matched adult psychiatric patients without ASD were selected for comparison. All patients were referred for psychiatric consultation. Socio-demographic variables were collected from the patients' record. Diagnosis of psychiatric disorders including ASD was made by an experienced psychiatrist. It was done clinically based on all available information, examination and relevant investigations. Diagnoses were assigned according to DSM-5. Then comparisons of psychiatric disorders were made between the two patient groups.

Result. The cases were ranged from 18-41 years with the mean of $26.72 \pm 6.5$ years. Among them, 22 were male and 14 were female. Male-female ratio was 1.6:1. Most of the subjects received no education and were from middle income family with urban background. Mean number of comorbid psychiatric disorders was 1.92 in patients with ASD and 1.67 in patients without
ASD and the difference was significant $(\mathrm{P}=0.04)$. Most two frequent comorbidities among ASD patients were Obsessive Compulsive Disorder (27.77\%) and Major Depressive Disorder (25\%) followed by Specific Phobia(19.44\%), Social Phobia and Intermittent Explosive Disorder(16.67\%) for each, Attention Deficit Hyperactivity Disorder(13.89\%) and Conduct Disorder $(11.11 \%)$. All these disorders were significantly higher than patients without ASD. Conversely, Major Depressive Disorder (30.55\%) was most frequent among the patients without ASD and that was even significantly higher than patients with ASD. Other frequent disorders like Bipolar Disorder, Schizophrenia, Generalized Anxiety Disorder and Substance Related Disorder were also higher among non-ASD patients.

Conclusion. This research shows that comorbid psychiatric disorders were frequently found in patients with ASD. Subsequent broad-based studies using extensive measures of psychopathology are required to confirm these preliminary findings. Greater understanding of the presence of other psychiatric disorders in ASD patients will turn this awareness into action.

\section{Bournewood in Belfast: who's ready?}

Hayley Bowes*, Joseph Kane and Gillian McPherson

Belfast Health and Social Care Trust

${ }^{\star}$ Corresponding author.

doi: $10.1192 /$ bjo.2021.640

Aims. We aimed to determine doctors' confidence in completing capacity assessments and reports associated with new Deprivation of Liberty Safeguards (DoLS), and the impact that these would have on practice. We hypothesised that psychiatrists would have greater confidence in completing the requisite assessments and would anticipate a greater impact on practice than doctors in other specialties.

Background. On the 2ndDecember 2019 DoLS became the first part of the Mental Capacity Act (Northern Ireland) 2016 to be implemented, believed to apply to 7500 individuals across Northern Ireland (NI). As arguably the most significant change in mental health legislation in NI since 1986, the Department of Health commissioned training for all clinicians.

Method. We conducted a cross-sectional survey among doctors working within psychiatry, general medicine, anaesthetics and surgery in Belfast Health and Social Care Trust prior to implementation. The survey comprised seven questions with a 10 -point Likert scale. Statistical analysis included Pearson's $\chi 2$ and Spearman's rank tests.

Result. 79 doctors in psychiatry and 25 in other medical specialties completed the survey.Respondents were moderately confident in completing capacity assessments (median 6 (3-9)) and medical reports (median 5 (1-9)). Those that had completed training $(\mathrm{n}=86 ; 83 \%)$ were significantly more confident in capacity assessment (median $7(2-10)$ vs $4(1-7) ; \chi 2(18)=36.8, \mathrm{p}<0.01)$ and medical report completion (median 5 (1-9) vs 1 (1-5); $\chi 2(16)=27.2, \mathrm{p}=.04)$ than those that had not $(\mathrm{n}=18 ; 17 \%)$. Psychiatrists had greater confidence in conducting capacity assessments (median 7(2-10)) than other doctors (median 5(1-9); $\chi 2(9)=18.2, p=0.04)$. No significant differences were observed between the two groups with respect to medical report completion, or anticipated impact on practice.

Respondents who most frequently conducted capacity assessments as part of their current practice anticipated higher degrees of impact on their individual practice ( $\mathrm{rs}=0.51, \mathrm{p}<0.01)$ and their service $(\mathrm{rs}=0.50, \mathrm{p}<0.01)$ 